\title{
ABSOLUTE STABILITY, CONDITIONAL STABILITY AND BIFURCATION IN KOLMOGOROV-TYPE PREDATOR-PREY SYSTEMS WITH DISCRETE DELAYS
}

BY

\author{
SHIGUI RUAN
}

Department of Mathematics and Statistics, Dalhousie University, Halifax, Nova Scotia, Canada

\begin{abstract}
The dynamics of delayed systems depend not only on the parameters describing the models but also on the time delays from the feedback. A delay system is absolutely stable if it is asymptotically stable for all values of the delays and conditionally stable if it is asymptotically stable for the delays in some intervals. In the latter case, the system could become unstable when the delays take some critical values and bifurcations may occur. We consider three classes of Kolmogorov-type predator-prey systems with discrete delays and study absolute stability, conditional stability and bifurcation of these systems from a global point of view on both the parameters and delays.
\end{abstract}

1. Introduction. Let $x(t)$ and $y(t)$ denote the population density of prey and predator at time $t$, respectively. The first predator-prey model with (distributed) delay was proposed by Volterra [43]. With the modification of Brelot [8], the model has the form

$$
\begin{aligned}
& \dot{x}(t)=x(t)\left[r_{1}-a_{11} x(t)-a_{12} \int_{-\infty}^{t} F(t-s) y(s) d s\right], \\
& \dot{y}(t)=y(t)\left[-r_{2}+a_{21} \int_{-\infty}^{t} G(t-s) x(s) d s-a_{22} y(t)\right],
\end{aligned}
$$

where $r_{i}$ and $a_{i j}(i, j=1,2)$ are positive constants; $F$ and $G$ are nonnegative continuous delay kernels defined and integrable on $[0, \infty)$. It is known that system (1.1) has a locally asymptotically stable steady state under some suitable assumptions on the parameters and delays. Further detailed study on stability and bifurcation of system (1.1) can be found in Cushing [13]. When $F(t-s)=\delta\left(t-s-\tau_{1}\right), G(t-s)=\delta\left(t-s-\tau_{2}\right)$, where $\delta$ is the delta function, $\tau_{1}$ and $\tau_{2}$ are positive constants, system (1.1) becomes the following Lotka-Volterra predator-prey model with discrete delays:

$$
\begin{aligned}
& \dot{x}(t)=x(t)\left[r_{1}-a_{11} x(t)-a_{12} y\left(t-\tau_{1}\right)\right], \\
& \dot{y}(t)=y(t)\left[-r_{2}+a_{21} x\left(t-\tau_{2}\right)-a_{22} y(t)\right] .
\end{aligned}
$$

Received May 20, 1999.

2000 Mathematics Subject Classification. Primary 34K20, 92D25.

Research supported by the Natural Science and Engineering Research Council of Canada. 
A special case of system (1.1) is the following model:

$$
\begin{aligned}
& \dot{x}(t)=x(t)\left[r_{1}-a_{11} x(t)-a_{12} y(t)\right], \\
& \dot{y}(t)=y(t)\left[-r_{2}+a_{21} \int_{-x}^{t} G(t-s) x(s) d s\right],
\end{aligned}
$$

which has been studied extensively; see Cushing [13], MacDonald [38], Dai [14], Farkas et al. [17], Stépán [42], etc. It is known that the time delay in (1.3) will destablize the otherwise stable equilibrium and cause fluctuations in the populations via Hopf bifurcations. The discrete delay version of system (1.3) is

$$
\begin{aligned}
& \dot{x}(t)=x(t)\left[r_{1}-a_{11} x(t)-a_{12} y(t)\right] . \\
& \dot{y}(t)=y(t)\left[-r_{2}+a_{21} x(t-\tau)\right] .
\end{aligned}
$$

which has been considered in Kuang [33] and Beretta and Kuang [4].

Assuming that in the absence of predators they prey's growth is governed by Hutchinson's ([32]) logistic equation. May [37] proposed the following predator-prey system:

$$
\begin{aligned}
& \dot{x}(t)=x(t)\left[r_{1}-a_{11} \int_{-x}^{t} F(t-s) x(s) d s-a_{12} y(t)\right], \\
& \dot{y}(t)=y(t)\left[-r_{2}+a_{21} x(t)-a_{22} y(t)\right] .
\end{aligned}
$$

Detailed bifurcation analysis of system (1.5) was carried out in Hassard, Kazarinoff and Wan [27]. It has been found that system (1.5) exhibits Hopf bifurcations when the time delay passes through a critical value. The discrete delay version of system (1.5) is

$$
\begin{aligned}
& \dot{x}(t)=x(t)\left[r_{1}-a_{11} x(t-\tau)-a_{12} y(t)\right] . \\
& \dot{y}(t)=y(t)\left[-r_{2}+a_{21} x(t)-a_{22} y(t)\right] .
\end{aligned}
$$

A brief discussion about system (1.6) can be found in May [37].

For most of the predator-prey systems with discrete delays such as (1.2), (1.4), and (1.6), the characteristic equation of the linearized system at a steady state is a transcendental polynomial equation of the following form:

$$
\lambda^{2}+p \lambda+r+(s \lambda+q) e^{-\lambda \tau}=0 .
$$

where $p, r, q, s$ are real numbers. It is known that the steady state is asymptotically stable if all roots of the characteristic equation (1.7) have negative real parts. For a nonlinear delay equation, there are two types of stabilities: absolute stability (independent of the delay) and conditional stability (depending on the delay).

Denote $R=(-\infty, \infty)$. Consider the following general nonlinear delay differential system:

$$
\dot{x}(t)=f\left(x(t), x\left(t-\tau_{1}\right), \ldots, x\left(t-\tau_{m}\right)\right),
$$

where $x \in R^{n}, \tau_{j} \geq 0(1 \leq j \leq m)$ are constants, $f: R^{\prime \prime} \times C^{\prime \prime} \rightarrow R^{\prime \prime}$ is smooth enough to guarantee the existence and uniqueness of solutions to (1.8) under the initial value condition

$$
x(\theta)=\phi(\theta) . \quad \theta \in[-\tau, 0],
$$

where $C=C\left([-\tau, 0], R^{\prime \prime}\right), \tau=\max _{1 \leq i \leq m}\left\{\tau_{i}\right\}$. Suppose $f\left(x^{*}, x^{*}, \ldots, x^{*}\right)=0$, that is. $x=x^{*}$ is a steady state of system (1.8). 
Definition 1.1. The steady state $x=x^{*}$ of system (1.8) is called absolutely stable (i.e., asymptotically stable independent of the delays) if it is asymptotically stable for all delays $\tau_{j} \geq 0(1 \leq j \leq m) . x=x^{*}$ is called conditionally stable (i.e., asymptotically stable depending on the delays) if it is asymptotically stable for $\tau_{j}(1 \leq j \leq m$ ) in some intervals, but not necessarily for all delays $\tau_{j} \geq 0(1 \leq j \leq m)$.

The linearized system of (1.8) at $x=x^{*}$ has the following form:

$$
\dot{X}(t)=A_{0} X(t)+\sum_{j=1}^{m} A_{j} X\left(t-\tau_{j}\right),
$$

where $X \in R^{n}$ and each $A_{j}(0 \leq j \leq m)$ is an $n \times n$ constant matrix. Thus, the steady state $x=x^{*}$ of system (1.8) is absolutely stable (conditionally stable) if the linearized system (1.10) is absolutely stable (conditionally stable).

The characteristic equation associated with system (1.10) takes the form:

$$
\operatorname{det}\left[\lambda I-A_{0}-\sum_{j=1}^{m} A_{j} e^{-\lambda \tau_{j}}\right]=0 .
$$

The location of the roots of some transcendental equations such as (1.11) has been studied by many researchers; see Baptistini and Táboas [1], Bellman and Cooke [3], Boese [5], Brauer [7], Cooke and van den Driessche [12], Cooke and Grossman [11], Huang [31], Mahaffy [36], Ruan and Wei [41] and the references therein. The following result, which was proved by Chin [10] for $m=1$ and by Datko [15] and Hale, Infante and Tsen [25] for $m \geq 1$, gives necessary and sufficient conditions for the absolute stability of system (1.10).

Lemma 1.2. The system (1.10) is absolutely stable if and only if

(i) $\operatorname{Re} \lambda\left(\sum_{j=0}^{m} A_{j}\right)<0$;

(ii) $\operatorname{det}\left[i \omega I-A_{0}-\sum_{j=1}^{m} A_{j} e^{-i \omega \tau_{j}}\right] \neq 0$ for all $\omega>0$.

Note that assumption (i) means that system (1.10) with $\tau_{j}=0(1 \leq j \leq m)$ is asymptotically stable and assumption (ii) means that $i \omega$ is not a root of Eq. (1.11). Thus, roughly speaking, Lemma 1.2 says that the delay system (1.10) is absolutely stable if and only if the corresponding ODE system is asymptotically stable and the characteristic equation (1.11) has no purely imaginary roots. Using Lemma 1.2, one could derive absolute stability conditions for some delayed predator-prey systems.

On the other hand, if assumption (ii) does not hold, that is, if the characteristic equation (1.11) has a pair of purely imaginary roots, say $\pm i \omega_{0}$, then system (1.10) is not absolutely stable but could be conditionally stable. Suppose $\omega_{0}$ is achieved when one of the delays, say $\tau_{1}$, reaches a value $\tau_{1}^{0}$. Hence, when $\tau_{1}<\tau_{1}^{0}$, the real parts of all roots of the characteristic equation (1.11) still remain negative and system (1.10) is conditionally stable. When $\tau_{1}=\tau_{1}^{0}$, the characteristic equation (1.11) has a pair of purely imaginary roots $\pm i \omega_{0}$ and system (1.10) loses its stability. By Rouché's theorem (Dieudonné [16]) and continuity, if the transversality condition holds at $\tau_{1}=\tau_{1}^{0}$, then when $\tau_{1}>\tau_{1}^{0}$, the characteristic equation (1.11) will have at least one root with positive real part and system (1.10) becomes unstable. Moreover, Hopf bifurcations occur, that is, a family of 
periodic solutions bifurcates from the steady state as $\tau_{1}$ passes through the critical value $\tau_{1}^{0}$.

The effect of time delays on stability of predator-prey systems has been investigated by many researchers; see the monographs of Cushing [13], Gopalsamy [24], Kuang [33], and MacDonald [38] and references cited therein. For various types of delayed predator-prey systems, many researchers have been interested either in studying absolute stability of these models (see Brauer [6], Gopalsamy [22], Ma [35], Nunney [40], etc.) or in determining conditional stability and bifurcation of these models (see Hastings [38], MacDonald [38], May [37], Nunney [39], etc.). However, there are few papers dealing with absolute stability, conditional stability and Hopf bifurcation altogether from a global point of view on the parameters and delays.

In this paper, we first find conditions on the coefficients of the characteristic equation (1.7) so that all its roots have negative real parts for all $\tau \geq 0$. Then we will determine the critical value $\tau_{0}$ so that when $\tau<\tau_{0}$ the real parts of all roots of Eq. (1.7) remain negative, when $\tau=\tau_{0}$ Eq. (1.7) has a pair of purely imaginary roots, and when $\tau>\tau_{0}$, Eq. (1.7) has at least one root with positive real part. We then apply the obtained results to analyze the absolute stability, conditional stability and Hopf bifurcation of some delayed predator-prey systems. First, we consider the delayed Kolmogorov-type predator-prey model

$$
\begin{aligned}
& \dot{x}(t)=x(t) f\left(x(t), y\left(t-\tau_{1}\right)\right), \\
& \dot{y}(t)=y(t) g\left(x\left(t-\tau_{2}\right), y(t)\right),
\end{aligned}
$$

which has system (1.2) as a special case, and find that the positive steady state is absolutely stable under some suitable assumptions on $f$ and $g$. For the following predatorprey model with a discrete delay

$$
\begin{aligned}
& \dot{x}(t)=x(t) f(x(t), y(t)), \\
& \dot{y}(t)=y(t) g(x(t-\tau), y(t)),
\end{aligned}
$$

which is more general than system (1.4), we find that the positive steady state is conditionally stable and the delay does cause instability and hence Hopf bifurcations occur. Finally, we apply the obtained results to the delayed predator-prey model

$$
\begin{aligned}
& \dot{x}(t)=x(t) f(x(t-\tau), y(t)), \\
& \dot{y}(t)=y(t) g(x(t), y(t)),
\end{aligned}
$$

which is a generalization of May's model (1.6). For different parameter values, the positive steady state of system (1.14) could be absolutely stable, conditionally stable or unstable.

2. The second-degree transcendental polynomials. In this section, we shall study the distributions of the roots of the characteristic equation. Consider the seconddegree transcendental polynomial equation

$$
\lambda^{2}+p \lambda+r+(s \lambda+q) e^{-\lambda \tau}=0,
$$


where $p, r, q, s$ are real numbers. When $\tau=0$, Eq. (2.1) becomes

$$
\lambda^{2}+(p+s) \lambda+(q+r)=0 .
$$

Assume that all roots of Eq. (2.2) have negative real parts, which is true if

$$
\begin{aligned}
& p+s>0 \\
& q+r>0 .
\end{aligned}
$$

We want to determine if the real part of some root increases to reach zero and eventually becomes positive as $\tau$ varies. If $i \omega$ is a root of Eq. (2.1), then

$$
-\omega^{2}+i p \omega+i s \omega(\cos \tau \omega+i \sin \tau \omega)+r+q(\cos \tau \omega+i \sin \tau \omega)=0 .
$$

Separating the real and imaginary parts, we have

$$
\begin{aligned}
-\omega^{2}+r & =-q \cos \tau \omega+s \omega \sin \tau \omega, \\
p \omega & =-s \omega \cos \tau \omega-q \sin \tau \omega .
\end{aligned}
$$

It follows that $\omega$ satisfies

$$
\omega^{4}-\left(s^{2}-p^{2}+2 r\right) \omega^{2}+\left(r^{2}-q^{2}\right)=0 .
$$

The two roots of Eq. (2.4) can be expressed as follows:

$$
\omega_{ \pm}^{2}=\frac{1}{2}\left(s^{2}-p^{2}+2 r\right) \pm \frac{1}{2}\left[\left(s^{2}-p^{2}+2 r\right)^{2}-4\left(r^{2}-q^{2}\right)\right]^{\frac{1}{2}} .
$$

Thus, if

$$
s^{2}-p^{2}+2 r<0 \text { and } r^{2}-q^{2}>0 \text { or }\left(s^{2}-p^{2}+2 r\right)^{2}<4\left(r^{2}-q^{2}\right),
$$

then none of $\omega_{+}^{2}$ and $\omega_{-}^{2}$ is positive, that is, Eq. (2.4) does not have positive roots. Therefore, characteristic equation (2.1) does not have purely imaginary roots. Since $\left(H_{1}\right)$ and $\left(H_{2}\right)$ ensure that all roots of Eq. (2.2) have negative real parts, by Rouché's theorem, it follows that the roots of Eq. (2.1) have negative real parts too. This can be summarized as follows.

LEMmA 2.1. If $\left(H_{1}\right)-\left(H_{3}\right)$ hold, then all roots of Eq. (2.1) have negative real parts for all $\tau \geq 0$.

On the other hand, if

$$
r^{2}-q^{2}<0 \text { or } s^{2}-p^{2}+2 r>0 \text { and }\left(s^{2}-p^{2}+2 r\right)^{2}=4\left(r^{2}-q^{2}\right),
$$

then Eq. (2.4) has a positive root $\omega_{+}^{2}$ and if

$$
r^{2}-q^{2}>0, s^{2}-p^{2}+2 r>0 \text { and }\left(s^{2}-p^{2}+2 r\right)^{2}>4\left(r^{2}-q^{2}\right),
$$

then Eq. (2.4) has two positive roots $\omega_{ \pm}^{2}$. In both cases, the characteristic equation (2.1) has purely imaginary roots when $\tau$ takes certain values. These critical values $\tau_{j}^{ \pm}$of $\tau$ can be determined from system (2.3), given by

$$
\tau_{j}^{ \pm}=\frac{1}{\omega_{ \pm}} \arccos \left\{\frac{q\left(\omega_{ \pm}^{2}-r\right)-p s \omega_{ \pm}^{2}}{s^{2} \omega_{ \pm}^{2}+q^{2}}\right\}+\frac{2 j \pi}{\omega_{ \pm}}, \quad j=0,1,2, \ldots .
$$

From the above analysis we have the following lemma. 
Lemma 2.2. (i) If $\left(H_{1}\right),\left(H_{2}\right)$ and $\left(H_{4}\right)$ hold and $\tau=\tau_{j}^{+}$, then Eq. (2.1) has a pair of purely imaginary roots $\pm i w_{+}$.

(ii) If $\left(H_{1}\right),\left(H_{2}\right)$ and $\left(H_{5}\right)$ hold and $\tau=\tau_{j}^{+}\left(\tau=\tau_{j}^{-}\right.$respectively), then Eq. (2.1) has a pair of imaginary roots $\pm i w_{+}\left( \pm i w_{-}\right.$respectively $)$.

Then we would expect that the real part of some root to Eq. (2.1) becomes positive when $\tau>\tau_{j}^{+}$and $\tau<\tau_{j}^{-}$. To see if it is the case, denote

$$
\lambda_{j}^{ \pm}=\alpha_{j}^{ \pm}(\tau)+i w_{j}^{ \pm}(\tau), \quad j=0,1,2, \ldots,
$$

the root of Eq. (2.1) satisfying

$$
\alpha_{j}^{ \pm}\left(\tau_{j}^{ \pm}\right)=0, \quad w_{j}^{ \pm}\left(\tau_{j}^{ \pm}\right)=\omega_{ \pm} .
$$

We can verify that the following transversality conditions hold:

$$
\frac{d}{d \tau} \operatorname{Re} \lambda_{j}^{+}\left(\tau_{j}^{+}\right)>0, \quad \frac{d}{d \tau} \operatorname{Re} \lambda_{j}^{-}\left(\tau_{j}^{-}\right)<0 .
$$

It follows that $\tau_{j}^{ \pm}$are bifurcation values. Thus, we have the following theorem about the distribution of the characteristic roots of Eq. (2.1).

TheOREM 2.3. Let $\tau_{j}^{ \pm}(j=0,1,2, \ldots)$ be defined by $(2.6)$.

(i) If $\left(H_{1}\right)-\left(H_{3}\right)$ hold, then all roots of Eq. (2.1) have negative real parts for all $\tau \geq 0$.

(ii) If $\left(H_{1}\right),\left(H_{2}\right)$ and $\left(H_{4}\right)$ hold, then when $\tau \in\left[0, \tau_{0}^{+}\right)$all roots of Eq. (2.1) have negative real parts, when $\tau=\tau_{0}^{+}$Eq. (2.1) has a pair of purely imaginary roots $\pm i \omega_{+}$, and when $\tau>\tau_{0}^{+}$Eq. (2.1) has at least one root with positive real part.

(iii) If $\left(H_{1}\right),\left(H_{2}\right)$ and $\left(H_{5}\right)$ hold, then there is a positive integer $k$ such that there are $k$ switches from stability to instability to stability; that is, when

$$
\tau \in\left[0, \tau_{0}^{+}\right],\left(\tau_{0}^{-}, \tau_{1}^{+}\right), \ldots,\left(\tau_{k-1}^{-}, \tau_{k}^{+}\right),
$$

all roots of Eq. (2.1) have negative real parts, and when

$$
\tau \in\left[\tau_{0}^{+}, \tau_{0}^{-}\right),\left[\tau_{1}^{+}, \tau_{1}^{-}\right), \ldots,\left[\tau_{k-1}^{+}, \tau_{k-1}^{-}\right) \quad \text { and } \quad \tau>\tau_{k}^{+},
$$

Eq. (2.1) has at least one root with positive real part.

REMARK 2.4. We should mention that the main part of Theorem 2.3 was obtained by Cooke and Grossman [11] in analyzing a general second-order equation with delayed friction and delayed restoring force. For other related work, see Baptistini and Táboas [1], Bellman and Cooke [3], Boese [5], Brauer [7], Cooke and van den Driessche [12], Cooke and Grossman [11], Huang [31], Mahaffy [36], etc.

3. Delayed Kolmogorov-type predator-prey systems. In this section, we shall use the results in Sec. 2 to study absolute stability, conditional stability, and bifurcation of three classes of Kolmogorov-type predator-prey systems with delay. Let $x(t)$ and $y(t)$ denote the population density of prey and predator at time $t$, respectively. 
3.1. Model I. We first consider the following Kolmogorov-type predator-prey model with discrete delays appearing in the inter-specific interaction terms of both equations:

$$
\begin{aligned}
& \dot{x}(t)=x(t) f\left(x(t), y\left(t-\tau_{1}\right)\right), \\
& \dot{y}(t)=y(t) g\left(x\left(t-\tau_{2}\right), y(t)\right),
\end{aligned}
$$

where $\tau_{i} \geq 0(i=1,2)$ is a constant. Denote $C=C([-\tau, 0], R)$, where $\tau=\max \left\{\tau_{1}, \tau_{2}\right\}$. Assume that $f: R \times C \rightarrow R$ and $g: C \times R \rightarrow R$ satisfy the following assumptions:

$\left(A_{1}\right)$ there exists a point $\left(x^{*}, y^{*}\right)$ with $x^{*}>0, y^{*}>0$ for which $f\left(x^{*}, y^{*}\right)=g\left(x^{*}, y^{*}\right)=0$;

$\left(A_{2}\right) f$ and $g$ are continuously differentiable such that

$$
\frac{\partial f}{\partial x}<0, \quad \frac{\partial f}{\partial y}<0, \quad \frac{\partial g}{\partial x}>0, \quad \frac{\partial g}{\partial y}<0 .
$$

The initial values of system (3.1) are

$$
x(\theta)=\phi(\theta) \geq 0, \quad y(\theta)=\psi(\theta) \geq 0, \quad \theta \in[-\tau, 0],
$$

where $\phi$ and $\psi$ are continuous functions.

Assumption $\left(A_{1}\right)$ ensures that $\left(x^{*}, y^{*}\right)$ is a positive equilibrium of system $(3.1) .\left(x^{*}, y^{*}\right)$ is called asymptotically stable if there exists a $\delta>0$ such that

$$
\sup _{-\tau \leq \theta \leq 0}\left[\left|\phi(\theta)-x^{*}\right|+\left|\psi(\theta)-y^{*}\right|\right]<\delta
$$

implies that

$$
\lim _{t \rightarrow \infty}(x(t), y(t))=\left(x^{*}, y^{*}\right)
$$

where $(x(t), y(t))$ is the solution of system (3.1) with initial values (3.2). $\left(x^{*}, y^{*}\right)$ is absolutely stable if it is asymptotically stable for all delays $\tau_{i} \geq 0(i=1,2)$ and is conditionally stable if it is asymptotically stable for $\tau_{i}(i=1,2)$ in some intervals. It is well known that the equilibrium $\left(x^{*}, y^{*}\right)$ of system $(3.1)$ is asymptotically stable if the zero equilibrium $(0,0)$ of the linearized system at $\left(x^{*}, y^{*}\right)$ is asymptotically stable. Let

$$
X(t)=x(t)-x^{*}, \quad Y(t)=y(t)-y^{*} .
$$

Then the linearized system at $\left(x^{*}, y^{*}\right)$ is

$$
\begin{aligned}
& \dot{X}(t)=\left(a x^{*}\right) X(t)+\left(b x^{*}\right) Y\left(t-\tau_{1}\right), \\
& \dot{Y}(t)=\left(c y^{*}\right) X\left(t-\tau_{2}\right)+\left(d y^{*}\right) Y(t),
\end{aligned}
$$

where

$$
a=\frac{\partial f}{\partial x}\left(x^{*}, y^{*}\right), \quad b=\frac{\partial f}{\partial y}\left(x^{*}, y^{*}\right), \quad c=\frac{\partial g}{\partial x}\left(x^{*}, y^{*}\right), \quad d=\frac{\partial g}{\partial y}\left(x^{*}, y^{*}\right) .
$$

The characteristic equation is

$$
\lambda^{2}-\left(a x^{*}+d y^{*}\right) \lambda+a d x^{*} y^{*}-b c x^{*} y^{*} e^{-\lambda\left(\tau_{1}+\tau_{2}\right)}=0 .
$$

Let $\tau=\tau_{1}+\tau_{2}$. Then it can be written as

$$
\lambda^{2}+p \lambda+r+q e^{-\lambda \tau}=0
$$

where

$$
p=-2\left(a x^{*}+d y^{*}\right)>0, \quad r=4 a d x^{*} y^{*}>0, \quad q=4 b c x^{*} y^{*}<0 .
$$


Equation (3.6) is a special case of Eq. (2.1) with $s=0$. Clearly, we can see that $\left(H_{1}\right)$ is satisfied. Since $a d-b c>0$, we have

$$
q+r=4(a d-b c) x^{*} y^{*}>0
$$

which implies that $\left(\mathrm{H}_{2}\right)$ is satisfied. We also have

$$
-p^{2}+2 r=-4\left(a^{2}\left(x^{*}\right)^{2}+d^{2}\left(y^{*}\right)^{2}\right)<0, \quad r^{2}-q^{2}=16\left(x^{*}\right)^{2}\left(y^{*}\right)^{2}(a d+b c)(a d-b c) .
$$

So $\left(H_{3}\right)$ is satisfied if $a d+b c>0$. By Theorem 2.3, all roots of the characteristic equation (3.6) have negative real parts if and only if $a d+b c>0$. Therefore, we have the following theorem.

Theorem 3.1. Suppose that $f$ and $g$ satisfy the assumptions $\left(A_{1}\right)$ and $\left(A_{2}\right)$. Then the positive equilibrium $\left(x^{*}, y^{*}\right)$ of the delayed predator-prey system (3.1) is absolutely stable (asymptotically stable for all $\tau_{i} \geq 0(i=1,2)$ ) if and only if $a d+b c>0$.

REMARK 3.2. System (3.1) with $\tau_{1}=\tau_{2}$ was studied by Gopalsamy [23] who showed that the delay is "harmless" in the sense that the positive steady state is asymptotically stable independent of the delay. Our result not only supports Gopalsamy's claim but also generalizes his to the case with two delays.

REMARK 3.3. There was a typo in the proof of Theorem 1 in [23]. Equation (2.19) should read

$$
L(a)=1 \pm r\left\{\left(a-q^{2}\right)^{2}+a^{2} p^{2}\right\}^{-\frac{1}{2}} .
$$

The negative sign in the power term was missing in [23] and the mistake was not corrected in the monograph of Gopalsamy [24] (p. 207, Eq. (3.5.17)).

As an example, consider the Lotka-Volterra predator-prey model with two discrete delays:

$$
\begin{aligned}
& \dot{x}(t)=x(t)\left[r_{1}-a_{11} x(t)-a_{12} y\left(t-\tau_{1}\right)\right], \\
& \dot{y}(t)=y(t)\left[-r_{2}+a_{21} x\left(t-\tau_{2}\right)-a_{22} y(t)\right],
\end{aligned}
$$

where $r_{i}, a_{i j}(i, j=1,2)$ are positive constants. If

$$
r_{1} a_{21}-r_{2} a_{11}>0
$$

then system (3.7) has a positive equilibrium $E^{*}=\left(x^{*}, y^{*}\right)$, where

$$
x^{*}=\frac{r_{1} a_{22}+r_{2} a_{12}}{a_{11} a_{22}+a_{12} a_{21}}, \quad y^{*}=\frac{r_{1} a_{21}-r_{2} a_{11}}{a_{11} a_{22}+a_{12} a_{21}} .
$$

The condition $a d+b c>0$ becomes $a_{11} a_{22}-a_{12} a_{21}>0$. Thus, by Theorem 3.1 , we have the following result on the stability of $E^{*}=\left(x^{*}, y^{*}\right)$.

Corollary 3.4. If condition (3.8) is satisfied, i.e., if the positive equilibrium $E^{*}=$ $\left(x^{*}, y^{*}\right)$ exists, then it is absolutely stable (asymptotically stable for all $\tau_{i} \geq 0(i=1,2)$ ) if and only if $a_{11} a_{22}-a_{12} a_{21}>0$.

REMARK 3.5. By the results in He [30] and Lu and Wang [34] we can see that the positive equilibrium $E^{*}=\left(x^{*}, y^{*}\right)$ of system (3.7) is indeed globally stable. 
3.2. Model II. Next we consider the following Kolmogorov-type predator-prey model with a discrete delay appearing in the inter-specific interaction term of the predator equation:

$$
\begin{aligned}
& \dot{x}(t)=x(t) f(x(t), y(t)), \\
& \dot{y}(t)=y(t) g(x(t-\tau), y(t)),
\end{aligned}
$$

where $\tau \geq 0$ is a constant, $C=C([-\tau, 0], R), f: R^{2} \rightarrow R$ and $g: C \times R \rightarrow R$ satisfy the assumptions $\left(A_{1}\right)$ and

$\left(A_{2}^{\prime}\right) f$ and $g$ are continuously differentiable such that

$$
\frac{\partial f}{\partial x}<0, \quad \frac{\partial f}{\partial y}<0, \quad \frac{\partial g}{\partial x}>0, \quad \frac{\partial g}{\partial y} \leq 0 .
$$

The initial values of system (3.10) are

$$
x(\theta)=\phi(\theta) \geq 0, \quad \theta \in[-\tau, 0], \quad y(0)=y_{0} \geq 0 .
$$

By the assumption $\left(A_{1}\right),\left(x^{*}, y^{*}\right)$ is a positive equilibrium of system (3.10). The stability of $\left(x^{*}, y^{*}\right)$ can be defined analogously as in Model I. Using (3.3), we have the linearized system:

$$
\begin{aligned}
& \dot{X}(t)=\left(a x^{*}\right) X(t)+\left(b x^{*}\right) Y(t), \\
& \dot{Y}(t)=\left(c y^{*}\right) X(t-\tau)+\left(d y^{*}\right) Y(t),
\end{aligned}
$$

where $a<0, b<0, c>0$, and $d \leq 0$ are defined in (3.5). The characteristic equation is

$$
\lambda^{2}-\left(a x^{*}+d y^{*}\right) \lambda+a d x^{*} y^{*}-b c x^{*} y^{*} e^{-\lambda \tau}=0 .
$$

Compared with (2.1), we have

$$
p=-\left(a x^{*}+d y^{*}\right)>0, \quad r=a d x^{*} y^{*} \geq 0, \quad s=0, \quad q=-b c x^{*} y^{*}>0 .
$$

Hence, conditions $\left(H_{1}\right)$ and $\left(H_{2}\right)$ are satisfied. Also,

$$
-p^{2}+2 r=-\left(a x^{*}\right)^{2}-\left(b x^{*}\right)^{2}<0, \quad r^{2}-q^{2}=(a d+b c)(a d-b c)\left(x^{*} y^{*}\right)^{2} .
$$

It follows that if $a d+b c>0$, then conditions $\left(H_{3}\right)$ hold; if $a d+b c<0$, then conditions $\left(H_{4}\right)$ hold. Denote

$$
\omega_{+}=\left\{\frac{1}{2}\left(2 r-p^{2}\right)+\left[\frac{1}{4}\left(2 r-p^{2}\right)^{2}-\left(r^{2}-q^{2}\right)\right]^{1 / 2}\right\}^{1 / 2}
$$

and

$$
\tau_{j}^{+}=\frac{1}{\omega_{+}} \arccos \left\{\frac{\omega_{+}^{2}-r}{q}\right\}+\frac{2 j \pi}{\omega_{+}}, \quad j=0,1,2, \ldots .
$$

By Theorem 2.3, we have the following theorem.

Theorem 3.6. Suppose that $f$ and $g$ satisfy the assumptions $\left(A_{1}\right)$ and $\left(A_{2}^{\prime}\right)$. Let $\omega_{+}$ and $\tau_{j}^{+}$be defined by (3.13) and (3.14), respectively.

(i) If $a d+b c>0$, then the positive equilibrium $\left(x^{*}, y^{*}\right)$ of the delayed predator-prey system (3.10) is absolutely stable.

(ii) If $a d+b c<0$, then $\left(x^{*}, y^{*}\right)$ of system (3.10) is conditionally stable: it is asymptotically stable when $\tau \in\left[0, \tau_{0}^{+}\right)$and unstable when $\tau>\tau_{0}^{+}$. Moreover, system (3.10) undergoes Hopf bifurcations at $\left(x^{*}, y^{*}\right)$ when $\tau=\tau_{j}^{+}(j=0,1,2, \ldots)$. 
As an example, consider the following delayed Lotka-Volterra type predator-prey model:

$$
\begin{aligned}
& \dot{x}(t)=x(t)\left[r_{1}-a_{11} x(t)-a_{12} y(t)\right], \\
& \dot{y}(t)=y(t)\left[-r_{2}+a_{21} x(t-\tau)\right],
\end{aligned}
$$

where $r_{1}, r_{2}, a_{11}, a_{12}$, and $a_{21}$ are positive constants. If (3.8) holds, then system (3.15) has a positive equilibrium $\left(x^{*}, y^{*}\right)$, where

$$
x^{*}=\frac{r_{2}}{a_{21}}, \quad y^{*}=\frac{r_{1} a_{21}-r_{2} a_{11}}{a_{12} a_{21}} .
$$

Since $d=0$, we have $a d+b c=-a_{12} a_{21}<0$ and

$$
p=a_{11} x^{*}>0, \quad r=0, \quad s=0, \quad q=a_{12} a_{21} x^{*} y^{*}>0 .
$$

Thus,

$$
\omega_{+}=\left\{-\frac{1}{2}\left(a_{11} x^{*}\right)^{2}+\left[\frac{1}{4}\left(\left(a_{11} x^{*}\right)^{4}+a_{12} a_{21} x^{*} y^{*}\right)^{2}\right]^{1 / 2}\right\}^{1 / 2}
$$

and

$$
\tau_{j}^{+}=\frac{1}{\omega_{+}} \arccos \left\{\frac{\omega_{+}^{2}}{a_{12} a_{21} x^{*} y^{*}}\right\}+\frac{2 j \pi}{\omega_{+}}, \quad j=0,1,2, \ldots .
$$

Corollary 3.7. Suppose (3.8) holds. Then the positive equilibrium $\left(x^{*}, y^{*}\right)$ of system (3.15) is conditionally stable: it is asymptotically stable when $\tau \in\left[0, \tau_{0}^{+}\right)$and unstable when $\tau>\tau_{0}^{+}$, where $\tau_{0}^{+}$is defined by (3.17). Moreover, when $\tau=\tau_{0}^{+}$a Hopf bifurcation occurs at $\left(x^{*}, y^{*}\right)$.

Remark 3.8. Corollary 3.7 can be derived from the analysis in Sec. 3.3 of Kuang [33]; see also Sec. 4 in Beretta and Kuang [4].

REMARK 3.9. Theorem 3.6 can be used to study stability and bifurcation in some other delayed predator-prey models which are in the form of system (3.10), such as the delayed predator-prey model with mutual interference analyzed in Cao and Freedman [9] and Freedman and Rao [19], the generalized Gause-type predator-prey model with delay studied by Zhao, Kuang and Smith [45], etc.

3.3. Model III. We consider the Kolmogorov-type predator-prey model with a discrete delay appearing in the intra-specific interaction term of the prey equation:

$$
\begin{aligned}
& \dot{x}(t)=x(t) f(x(t-\tau), y(t)), \\
& \dot{y}(t)=y(t) g(x(t), y(t)),
\end{aligned}
$$

where $\tau \geq 0$ is a constant, $C=C([-\tau, 0], R), f: C \times R \rightarrow R$ and $g: R^{2} \rightarrow R$ satisfy the assumptions $\left(A_{1}\right)$ and $\left(A_{2}\right)$. The initial values of system (3.18) are

$$
x(\theta)=\phi(\theta) \geq 0, \quad \theta \in[-\tau, 0], \quad y(0)=y_{0} \geq 0 .
$$

System (3.18) has the same positive equilibrium $\left(x^{*}, y^{*}\right)$ as system (3.1). Using the change of variables (3.3), the linearized system of $(3.18)$ at $\left(x^{*}, y^{*}\right)$ is

$$
\begin{aligned}
& \dot{X}(t)=\left(a x^{*}\right) X(t-\tau)+\left(b x^{*}\right) Y(t), \\
& \dot{Y}(t)=\left(c y^{*}\right) X(t)+\left(d y^{*}\right) Y(t),
\end{aligned}
$$


where $a<0, b<0, c>0$, and $d<0$ are defined by (3.5). The characteristic equation of the linear system (3.19) has the form:

$$
\lambda^{2}-d y^{*} \lambda-b c x^{*} y^{*}+\left(-a x^{*} \lambda+a d x^{*} y^{*}\right) e^{-\lambda \tau}=0 .
$$

It is of the form of Eq. (2.1) with

$$
p=-d y^{*}, \quad r=-b c x^{*} y^{*}, \quad s=-a x^{*}, \quad q=a d x^{*} y^{*} .
$$

Clearly, $\left(H_{1}\right)$ and $\left(H_{2}\right)$ hold. If we assume that

$$
s^{2}-p^{2}+2 r=\left(a x^{*}\right)^{2}-\left(d y^{*}\right)^{2}+2 b c x^{*} y^{*}<0
$$

and

$$
a d+b c<0
$$

then

$$
r^{2}-q^{2}=-(a d+b c)(a d-b c)\left(x^{*} y^{*}\right)^{2}>0
$$

by assumption $\left(A_{2}\right)$, that is, $\left(H_{3}\right)$ is satisfied. Therefore, all roots of the characteristic equation (3.20) have negative real parts.

Theorem 3.10. Suppose that $f$ and $g$ satisfy the assumptions $\left(A_{1}\right)$ and $\left(A_{2}\right)$. If conditions (3.21) and (3.22) are satisfied, then the positive equilibrium $\left(x^{*}, y^{*}\right)$ of system (3.18) is absolutely stable.

If we assume that

$$
a d+b c>0
$$

then

$$
r^{2}-q^{2}=-(a d+b c)(a d-b c)\left(x^{*} y^{*}\right)^{2}<0
$$

and $\left(H_{4}\right)$ is satisfied. It follows that the characteristic equation (3.20) has a pair of purely imaginary roots $\pm i \omega_{+}$, where

$$
\begin{aligned}
\omega_{+}=\left\{\frac { 1 } { 2 } \left[\left(a x^{*}\right)^{2}-\left(d y^{*}\right)^{2}-2 b c x^{*} y^{*}\right.\right. \\
\left.\quad+\left(\left(\left(a x^{*}\right)^{2}-\left(d y^{*}\right)^{2}-2 b c x^{*} y^{*}\right)^{2}-4\left(b^{2} c^{2}-a^{2} d^{2}\right)\left(x^{*} y^{*}\right)^{2}\right)^{1 / 2}\right\}^{1 / 2} .
\end{aligned}
$$

Denote

$$
\tau_{j}^{+}=\frac{1}{\omega_{+}} \arccos \left\{\frac{b c d\left(y^{*}\right)^{2}}{a\left[\omega_{+}^{2}+\left(d y^{*}\right)^{2}\right]}\right\}+\frac{2 j \pi}{\omega_{+}}, \quad j=0,1,2, \ldots
$$

By Theorem 2.3, we have the following result on the stability of the positive equilibrium $\left(x^{*}, y^{*}\right)$ of system $(3.18)$.

TheOREm 3.11. Suppose that $f$ and $g$ satisfy the assumptions $\left(A_{1}\right)$ and $\left(A_{2}\right)$ and that condition (3.23) holds. Let $\omega_{+}$and $\tau_{j}^{+}(j=0,1,2, \ldots)$ be defined by (3.24) and (3.25), respectively. Then the steady state $\left(x^{*}, y^{*}\right)$ of system (3.18) is conditionally stable. More precisely, we have

(i) if $\tau \in\left[0, \tau_{0}^{+}\right)$, then $\left(x^{*}, y^{*}\right)$ is asymptotically stable;

(ii) if $\tau>\tau_{0}^{+}$, then $\left(x^{*}, y^{*}\right)$ is unstable; 
(iii) if $\tau=\tau_{j}^{+}(j=0,1,2, \ldots)$, then system (3.18) undergoes Hopf bifurcations at $\left(x^{*}, y^{*}\right)$.

Finally, if we assume that condition (3.22),

$$
s^{2}-p^{2}+2 r=\left(a x^{*}\right)^{2}-\left(d y^{*}\right)^{2}+2 b c x^{*} y^{*}>0,
$$

and

$$
\begin{aligned}
& \left(s^{2}+s r-p^{2}\right)^{2}-4\left(r^{2}-q^{2}\right) \\
& \quad=\left[\left(a x^{*}\right)^{2}-\left(d y^{*}\right)^{2}+2 b c x^{*} y^{*}\right]^{2}+(a d+b c)(a d-b c)\left(x^{*} y^{*}\right)^{2}>0
\end{aligned}
$$

hold, then the characteristic equation (3.20) has two pairs of purely imaginary roots $\pm i \omega_{ \pm}$, where $\omega_{+}$is given in (3.24) and $\omega_{-}$is defined as follows:

$$
\begin{aligned}
\omega_{-}=\{ & \frac{1}{2}\left[\left(a x^{*}\right)^{2}-\left(d y^{*}\right)^{2}-2 b c x^{*} y^{*}\right. \\
& \left.\left.-\left(\left(\left(a x^{*}\right)^{2}-\left(d y^{*}\right)^{2}-2 b c x^{*} y^{*}\right)^{2}-4\left(b^{2} c^{2}-a^{2} d^{2}\right)\left(x^{*} y^{*}\right)^{2}\right)^{1 / 2}\right]\right\}^{1 / 2} .
\end{aligned}
$$

Denote

$$
\tau_{j}^{-}=\frac{1}{\omega_{-}} \arccos \left\{\frac{b c d\left(y^{*}\right)^{2}}{a\left[\omega_{-}^{2}+\left(d y^{*}\right)^{2}\right]}\right\}+\frac{2 j \pi}{\omega_{-}}, \quad j=0,1,2, \ldots .
$$

Again by Theorem 2.3, we have the following theorem on the switching of stability in system (3.18).

THEOREM 3.12. Suppose that $f$ and $g$ satisfy the assumptions $\left(A_{1}\right)$ and $\left(A_{2}\right)$ and that conditions $(3.22),(3.26)$, and $(3.27)$ hold. Let $\omega_{+}$and $\tau_{j}^{+}(j=0,1,2, \ldots)$ be defined by (3.24) and (3.25), respectively, and $\omega_{-}$and $\tau_{j}^{-}(j=0,1,2, \ldots)$ be defined by (3.28) and (3.29), respectively. Then there is a positive integer $k$ such that when $\tau \in\left[0, \tau_{0}^{+}\right)$, $\left(\tau_{0}^{-}, \tau_{1}^{+}\right), \ldots,\left(\tau_{k-1}^{-}, \tau_{k}^{+}\right)$, the positive equilibrium $\left(x^{*}, y^{*}\right)$ of system $(3.18)$ is asymptotically stable; when $\tau \in\left[0, \tau_{0}^{+}\right),\left(\tau_{0}^{-}, \tau_{1}^{+}\right), \ldots,\left(\tau_{k-1}^{-}, \tau_{k}^{+}\right)$and $\tau>\tau_{k}^{+},\left(x^{*}, y^{*}\right)$ is unstable.

As an example, consider the delayed Lotka-Volterra predator-prey model proposed by May [37]:

$$
\begin{aligned}
& \dot{x}(t)=x(t)\left[r_{1}-a_{11} x(t-\tau)-a_{12} y(t)\right], \\
& \dot{y}(t)=y(t)\left[-r_{2}+a_{21} x(t)-a_{22} y(t)\right],
\end{aligned}
$$

where $r_{i}, a_{i j}(i, j=1,2)$ are positive constants. If (3.8) holds, then system (3.30) has a positive equilibrium $\left(x^{*}, y^{*}\right)$ given by (3.9). Condition $a d+b c>0$ again becomes $a_{11} a_{22}-a_{12} a_{21}>0$. Also,

$$
\begin{aligned}
\omega_{ \pm}=\{ & \frac{1}{2}\left[\left(a_{11} x^{*}\right)^{2}-\left(a_{22} y^{*}\right)^{2}-2 a_{12} a_{21} x^{*} y^{*}\right. \\
& \left.\left.\left. \pm\left[\left(a_{11} x^{*}\right)^{2}+\left(a_{22} y^{*}\right)^{2}\right)^{2}-4\left(\left(a_{11} x^{*}\right)^{2}-\left(a_{22} y^{*}\right)^{2}\right) a_{12} a_{21} x^{*} y^{*}\right]\right]^{1 / 2}\right\}^{1 / 2}
\end{aligned}
$$

and

$$
\tau_{j}^{ \pm}=\frac{1}{\omega_{ \pm}} \arccos \left\{\frac{-a_{22}\left(y^{*}\right)^{2}}{a_{11}\left[\omega_{ \pm}^{2}+\left(a_{22} y^{*}\right)^{2}\right]}\right\}+\frac{2 j \pi}{\omega_{ \pm}}, \quad j=0,1,2, \ldots
$$

We have the following result on the stability of system (3.30).

Corollary 3.13. Let $\omega_{ \pm}$and $\tau_{j}^{ \pm}$be defined by (3.31) and (3.32), respectively. 
(i) If $\left(a_{11} x^{*}\right)^{2}-\left(a_{22} y^{*}\right)^{2}+2 a_{12} a_{21} x^{*} y^{*}<0$ and $a_{11} a_{22}-a_{12} a_{21}<0$, then the equilibrium $\left(x^{*}, y^{*}\right)$ of system (3.30) is absolutely stable.

(ii) If $a_{11} a_{22}-a_{12} a_{21}>0$, then $\left(x^{*}, y^{*}\right)$ is conditionally stable: it is asymptotically stable when $\tau \in\left[0, \tau_{0}^{+}\right)$and unstable when $\tau>\tau_{0}^{+}$. A Hopf bifurcation occurs when $\tau=\tau_{0}^{+}$.

(iii) If $\left(a_{11} x^{*}\right)^{2}-\left(a_{22} y^{*}\right)^{2}+2 a_{12} a_{21} x^{*} y^{*}>0$ and $a_{11} a_{22}-a_{12} a_{21}<0$, then there is a positive integer $k$, such that the equilibrium $\left(x^{*}, y^{*}\right)$ switches $k$ times from stability to instability to stability; that is, $\left(x^{*}, y^{*}\right)$ is asymptotically stable when

$$
\tau \in\left[0, \tau_{0}^{+}\right),\left(\tau_{0}^{-}, \tau_{1}^{+}\right), \ldots,\left(\tau_{k-1}^{-}, \tau_{k}^{+}\right)
$$

and unstable when

$$
\tau \in\left[\tau_{0}^{+}, \tau_{0}^{-}\right),\left[\tau_{1}^{+}, \tau_{1}^{-}\right), \ldots,\left[\tau_{k-1}^{+}, \tau_{k-1}^{-}\right) \quad \text { and } \quad \tau>\tau_{k}^{+} .
$$

4. Discussion. We have studied absolute stability, conditional stability, and bifurcation in three classes of Kolmogorov-type predator-prey systems with discrete delays. For the first type of models, there are two delays in the inter-specific interaction terms of both equations. It has been shown that the positive steady state of such a system is absolutely stable under some assumptions on the interaction terms. In the second type of models, a delay appears only in the inter-specific interaction term of the predator equation. We have seen that the system is only conditionally stable for the delay in some interval and Hopf bifurcations could occur when the delay takes some critical values. The third type of model is a generalization of May's predator-prey model in which a delay appears in the intra-specific interaction term of the prey equation. It is very interesting that under various assumptions on the interaction terms, the positive steady state of this type of models could be absolutely stable, conditionally stable, and unstable. Moreover, when the delay varies, the steady state switches from stability to instability a finite number of times.

The delayed predator-prey systems we have studied are all of the Kolmogorov-type. Bartlett [2] and Wangersky and Cunningham [44] were the first to propose delayed predator-prey models that are not of the Kolmogorov-type (in fact, they were the first to incorporate discrete delays into predator-prey models). Bartlett assumed that there is a time lag $\tau_{1}$ in the growth to maturity of the prey, and similarly there is a $\tau_{2}$ for the predators. He then modified the Lotka-Volterra system into the following form:

$$
\begin{aligned}
& \dot{x}(t)=r_{1} x\left(t-\tau_{1}\right)-a_{12} x(t) y(t), \\
& \dot{y}(t)=-r_{2} y(t)+a_{21} x\left(t-\tau_{2}\right) y\left(t-\tau_{2}\right) .
\end{aligned}
$$

Wangersky and Cunningham's model is relatively more well known in the literature but is a special case of (4.1), having the form

$$
\begin{aligned}
& \dot{x}(t)=x(t)\left[r_{1}-a_{11} x(t)-a_{12} y(t)\right], \\
& \dot{y}(t)=-r_{2} y(t)+a_{21} x(t-\tau) y(t-\tau) .
\end{aligned}
$$

This model means that a duration of $\tau$ time units elapses when an individual prey is killed and the moment when the corresponding addition is made to the predator population. Wangersky and Cunningham [44] briefly analyzed their model (4.2), Goel 
et al. [21] pointed out that there are problems in Wangersky and Cunningham's analysis and reconsidered the model, but their own analysis is also incomplete (see Nunney [40]). Some special cases of Bartlett's model (4.1) when $\tau_{1}=0$, or $\tau_{2}=0$, or $\tau_{1}=\tau_{2}$ have been studied by Hastings [28], Nunney [39, 40], Ma [35], etc. Local stability analysis of Bartlett's model (4.1) was carried out in Hastings [29].

Using the techniques of this paper, we can show that, under certain assumptions on the parameters, Wangersky and Cunningham's model (4.2) is absolutely stable (for all delays); under another set of conditions on the parameters the model is conditionally stable (for the delay in some intervals); when the delay takes some critical values the system becomes unstable and bifurcations occur. It will be very interesting to determine the detailed dynamics of the two-delay Bartlett model (4.1). We leave this for future considerations.

\section{REFERENCES}

[1] M. Baptistini and P. Táboas, On the stability of some exponential polynomials, J. Math. Anal. Appl. 205, 259-272 (1997)

[2] M. S. Bartlett, On theoretical models for competitive and predatory biological systems, Biometrika 44, $27-42$ (1957)

[3] R. Bellman and K. L. Cooke, Differential-Difference Equations, Academic Press, New York, 1963

[4] E. Beretta and Y. Kuang, Convergence results in a well-known delayed predator-prey system, J. Math. Anal. Appl. 204, 840 853 (1996)

[5] F. G. Boese, Stability criteria for second-order dynamical systems involving several time delays, SIAM J. Math. Anal. 26, 13061330 (1995)

[6] F. Brauer, Characteristic return times for harvested population models with time lag, Math. Biosci. 45, 295-311 (1979)

[7] F. Brauer, Absolute stability in delay equations, J. Differential Equations 69, 185-191 (1987)

[8] M. Brelot, Sur le problème biologique héréditaire de deux espèces dévorante et dévoré, Ann. Mat. Pura Appl. 9, 58-74 (1931)

[9] Y. Cao and H. I. Freedman, Global attractivity in time-delayed predator-prey systems, J. Austral. Math. Soc. Ser. B 38, 149-162 (1996)

[10] Y.-S. Chin, Unconditional stability of systems with time-lags, Acta Math. Sinica 1, 125-142 (1960)

[11] K. L. Cooke and Z. Grossman, Discrete delay, distributed delay and stability switches, J. Math. Anal. Appl. 86, 592-627 (1982)

[12] K. L. Cooke and P. van den Driessche, On zeros of some transcendental equations, Funkcialaj Ekvacioj 29, 77 -90 (1986)

[13] J. M. Cushing, Integrodifferential Equations and Delay Models in Population Dynamics, SpringerVerlag, Heidelberg, 1977

[14] L. S. Dai, Nonconstant periodic solutions in predator-prey systems with continuous time delay, Math. Biosci. 53, 149- 157 (1981)

[15] R. Datko, A procedure for determination of the exponential stability of certain differential difference equations, Quart. Appl. Math. 36, 279-292 (1978)

[16] J. Dieudonné, Foundations of Modern Analysis, Academic Press, New York, 1960

[17] A. Farkas, M. Farkas, and G. Szabó, Multiparameter bifurcation diagrams in predator-prey models with time lag, J. Math. Biol. 26, 93-103 (1988)

[18] H. I. Freedman and K. Gopalsamy, Nonoccurrence of stability switching in systems with discrete delays, Canad. Math. Bull. 31, $52-58$ (1988)

[19] H. I. Freedman and V. S. H. Rao, The tradeoff between mutual interference and time lags in predator-prey systems, Bull. Math. Biol. 45, 991-1004 (1983)

[20] H. I. Freedman and V. S. H. Rao, Stability criteria for a system involving two time delays, SIAM J. Appl. Anal. 46, 552-560 (1986)

[21] N. S. Goel, S. C. Maitra, and E. W. Montroll, On the Volterra and other nonlinear models of interacting populations, Rev. Modern Phys. 43, 231-276 (1971)

[22] K. Gopalsamy, Harmless delay in model systems, Bull. Math. Biol. 45, 295-309 (1983) 
[23] K. Gopalsamy, Delayed responses and stability in two-species systems, J. Austral. Math. Soc. Ser. B 25, 473-500 (1984)

[24] K. Gopalsamy, Stability and Oscillations in Delay Differential Equations of Population Dynamics, Kluwer Academic, Dordrecht, 1992

[25] J. K. Hale, E. F. Infante, and F.-S. P. Tsen, Stability in linear delay equations, J. Math. Anal. Appl. 105, 533-555 (1985)

[26] J. K. Hale and S. M. Verduyn Lunel, Introduction to Functional Differential Equations, SpringerVerlag, New York, 1993

[27] B. D. Hassard, N. D. Kazarinoff, and Y.-H. Wan, Theory and Applications of Hopf Bifurcation, Cambridge University Press, London, 1981

[28] A. Hastings, Age-dependent predation is not a simple process: I. Continuous time models, Theoret. Pop. Biol. 23, 347-362 (1983)

[29] A. Hastings, Delays in recruitment at different trophic levels: Effects on stability, J. Math. Biol. 21, 35-44 (1984)

[30] X.-Z. He, Stability and delays in a predator-prey system, J. Math. Anal. Appl. 198, 355-370 (1996)

[31] W. Huang, Algebraic criteria on the stability of the zero solutions of the second order delay differential equations, J. Anhui University, 1-7 (1985)

[32] G. E. Hutchinson, Circular cause systems in ecology, Ann. New York Acad. Sci. 50, 221-246 (1948)

[33] Y. Kuang, Delay Differential Equations with Applications in Population Dynamics, Academic Press, New York, 1993

[34] Z. Lu and W. Wang, Global stability for two-species Lotka-Volterra systems with delay, J. Math. Anal. Appl. 208, 277-280 (1997)

[35] Z. Ma, Stability of predation models with time delay, Applicable Analysis 22, 169-192 (1986)

[36] J. M. Mahaffy, A test for stability of linear differential delay equations, Quart. Appl. Math. 40, 193-202 (1982)

[37] R. M. May, Time delay versus stability in population models with two and three trophic levels, Ecology 4, 315-325 (1973)

[38] N. MacDonald, Time Lags in Biological Models, Springer-Verlag, Heidelberg, 1978

[39] L. Nunney, The effect of long time delays in predator-prey systems, Theoret. Pop. Biol. 27, 202-221 (1985)

[40] L. Nunney, Absolute stability in predator-prey models, Theoret. Pop. Biol. 28, 209-232 (1985)

[41] S. Ruan and J. Wei, On the zeros of transcendental functions with applications to stability of delay differential equations, preprint.

[42] G. Stépán, Great delay in a predator-prey model, Nonlinear Analysis 10, 913-929 (1986)

[43] V. Volterra, Leçons sur la théorie mathématique de la lutte pour la vie, Gauthier-Villars, Paris, 1931

[44] P. J. Wangersky and W. J. Cunningham, Time lag in prey-predator population models, Ecology 38, 136-139 (1957)

[45] T. Zhao, Y. Kuang, and H. L. Smith, Global existence of periodic solutions in a class of delayed Gause-type predator-prey systems, Nonlinear Analysis 28, 1373-1394 (1997) 